\title{
On the Giant Nano-subpulses in the Crab Pulsar
}

\author{
J. Gil \& George I. Melikidze \\ Institute of Astronomy, University of Zielona Góra, Poland
}

\begin{abstract}
Extremely short and powerful subpulses were recently detected within the giant radio pulses from the Crab pulsar. We argue that these are consistent with beaming due to the curvature of field lines, and thus with coherent curvature radiation. We also demonstrate that the observed fluxes are consistent with the emission of charged relativistic solitons, generated by means of modulational instability of the Langmuir turbulence associated with sparking discharge of the pulsar's polar gap.
\end{abstract}

\section{Introduction}

Although only four pulsars are known to emit giant pulses, understanding the mechanism of their radiation can lead to understanding the longstanding problem of pulsar radio emission. Extremely short and powerful, 2-ns, 1000-Jy, subpulses were recently detected within the giant pulses from the Crab pulsar. We here present a framework for understanding this phenomenon in terms of coherent curvature radiation, and the emission of charged relativistic solitons.

\section{The Model}

Let us consider a number of localized (point-like) sources of the coherent curvature radiation, moving relativistically (with Lorentz factor $\gamma=\left(1-\beta^{2}\right)^{-1 / 2}$, where $\beta=v / c \sim 1$ ) along a narrow bundle of dipolar magnetic field lines with a radius of curvature $\rho=1.8 r_{6}^{1 / 2} s^{-1} 10^{7} \mathrm{~cm}$, where $s$ is the normalized polar coordinate of the central line of the bundle, $r_{6}=r / R$ is the normalized emission altitude (for the frequency $\nu=5 \mathrm{GHz}, 5<r_{6}<35$ in the Crab pulsar; Kijak \& Gil 1998). The longitudinal source dimension is $\delta l$ and the distance between the sources is $\Delta l$. The curvature radiation is emitted along dipolar field lines into a narrow cone with an opening angle $\theta=1 / \gamma$. We also assume that other bundles carrying sources of coherent curvature radiation are separated by at least $\theta$, thus only one field line (narrow bundle) contributes to the radiation observed at a given rotational phase $\varphi=2 \pi t / P$, where $t$ is the intrapulse time. For a fixed arrangement of the observer's direction, the field line polar coordinate $s$ and the frequency-dependent emission altitude $r_{6}(\nu)$, each source is aligned (within $1 / \gamma$ ) with the observer during the time interval $\delta t=l / v=\rho \theta / v=\rho \gamma^{-1} / v$. Therefore, a continuous stream of relativistic sources moving along a curved trajectory would illuminate the observer during the time interval

$$
\Delta t=\rho \gamma^{-1} / c,
$$


provided that this "alignment" time scale is longer than the conventional angular beaming time scale $\Delta t^{\prime}=\gamma^{-1} P / 2 \pi$ related purely to rotation, that is, $\rho>$ $1.6 \times 10^{8} \mathrm{~cm}$ in the case of the Crab pulsar. Hankins et al. (2003) concluded that conventional angular beaming cannot explain the 2-ns duration of their subpulses, since it requires $\gamma \sim 10^{6}$, much above the expected $\gamma<1000$. Since, accidentally, for the Crab pulsar $\Delta t^{\prime} \sim \Delta t$, their conclusion applies also to the alignment time scale related to the field line curvature (Eq. [1]).

However, the situation can be quite different if the stream is not continuous but one is distinguished by emitting slightly more power $P$ than the average. Consider two particular points along a given dipolar field line: where the source becomes aligned, and then misaligned with the observer, respectively. The source covers a distance $l=\rho / \gamma$ between these points during an interval $\delta t=\rho \gamma^{-1} / v$. During this time the radiation emitted at the first alignment point travels a distance $L=c \delta t=(c / v) \rho \gamma^{-1}=\rho /(\beta \gamma)$. Thus, the radiation overtakes the source only by $\Delta=L-l=(1 / \beta-1)(\rho / \gamma) \approx \rho / \gamma^{3}$. This is the pulse length in space and thus the duration of the observed impulse emitted by the source is

$$
\Delta \tau=\frac{\Delta}{c}=\frac{\rho}{c \gamma^{3}}=\gamma^{-2} \Delta t
$$

where $\Delta t$ is the alignment time scale (Eq. [1]). Therefore, if the source dimension $\delta l \leq \Delta \approx \rho / \gamma^{3}$, then the apparent duration of the impulse $\Delta \tau$ is $\gamma^{2}$ times shorter than the actual time of source-observer alignment. Note that this apparent shortening effect has nothing to do with special relativity (time dilation) and/or Doppler effects, as both $\Delta t$ and $\Delta \tau$ are referred to the same observer's frame.

Both isolated 2-ns giant subpulses, as well as a sequence of a number of such subpulses (which can probably be called a giant pulse) can be seen in Figure 1 of Hankins et al. (2003). Within our model such a case would correspond to a sequence of sources, each having a longitudinal dimension $\delta l<\Delta$ and separation between adjacent sources $\Delta l>\Delta$, where $\Delta \approx \rho / \gamma^{3}$ is the spatial length of the impulse associated with each source. For each subpulse detected by Hankins et al. (2003) the duration $\Delta \tau=2 \times 10^{-9} \mathrm{~s}$ (Eq. [2]), which leads to

$$
\rho_{8} \approx 0.6 \gamma_{2}^{3}
$$

where $\rho_{8}=\rho / 10^{8} \mathrm{~cm}$ and $\gamma_{2}=\gamma / 10^{2}$.

As reported by Hankins et al. (2003), the ultra-short nano-giant subpulses detected at $\nu=5 \mathrm{GHz}$ in the Crab pulsar often exceed fluxes $S=1000 \mathrm{Jy}=$ $10^{-20} \mathrm{ergs} \mathrm{s}^{-1} \mathrm{~Hz}^{-1} \mathrm{~cm}^{-2}$. These fluxes can be converted into brightness temperature $T_{b}=S D^{2} /\left[2 k(\nu W)^{2}\right]$, where $D=2 \mathrm{kpc}=6 \times 10^{21} \mathrm{~cm}$ is the distance to the pulsar, $k$ is the Boltzmann constant and $W$ is the time scale of the corresponding emission process. Assuming that $W=2 \times 10^{-9} \mathrm{~s}$, one can obtain extraordinarily high brightness temperatures $T_{b} \sim 10^{38} \mathrm{~K}$, implying by far the most luminous emission from any astronomical object. However, within our model the actual emission process corresponding to a giant subpulse of duration $\Delta \tau=2 \times 10^{-9} \mathrm{~s}$ occurs over a much longer "alignment" time interval $W=\Delta t=\gamma^{2} \Delta \tau$ (Eqs. [1] and [2]), which leads to $\gamma^{4}$ times lower brightness temperatures. Since $\gamma$ is of the order of 100 , then $T_{b}<10^{30} \mathrm{~K}$, consistent with normal giant pulses in the Crab pulsar and other pulsars (see Fig. 1 in McLaughlin \& Cordes 2003). 
Let us consider a source(s) moving along a dipolar field line and emitting coherent curvature radiation with intrinsic power $P$. While moving over the alignment distance $l=\rho \gamma^{-1}$, the emitted energy $E=P \Delta t$, where the alignment interval $\Delta t=\rho \gamma^{-1} / c$ (Eq. [1]). This energy is received by the observer in a much shorter time $\Delta \tau=\gamma^{-2} \Delta t$ (Eq. [2]), and therefore the apparent luminosity

$$
\mathcal{L}=\frac{E}{\Delta \tau}=\gamma^{2} P
$$

Thus, the nanosecond giant subpulses appear $\gamma^{2}=\gamma_{2}^{2} 10^{2}$ times more luminous than the intrinsic power of their source(s). Also the apparent fluxes $S \propto \mathcal{L}$ are $\gamma^{2}$ times overestimated. It is important to emphasize that this apparent brightening is a direct consequence of the apparent shortening of the impulse duration described by Eq. (2) and has nothing to do with special relativity.

The apparent fluxes $S \sim 1000 \mathrm{Jy}$ of the nanosecond giant subpulses can be formally converted into the emitted luminosity $\mathcal{L}=S \theta^{2} D^{2} \Delta \nu$. With $D=2 \mathrm{kpc}$, $\theta=\gamma_{2}^{-1} 10^{-2}$ and $\Delta \nu \sim 10^{9} \mathrm{~Hz}$ one obtains $\mathcal{L} \sim \gamma_{2}^{-2} 10^{28} \mathrm{ergs} \mathrm{s}^{-1}$. This is an extremely large value, comparable with the total radio luminosity of the Crab pulsar $L_{R}=3.5 \times 10^{25+x}$ ergs $\mathrm{s}^{-1}$, where $x=\log L$ in $\mathrm{mJy}_{\mathrm{kpc}}^{2}$ from the pulsar catalog. The existence of such powerful localized sources of coherent radio emission seems questionable (of course, this argument is not independent from the extraordinarily high brightness temperatures $T_{b} \sim 10^{38} \mathrm{~K}$ derived above). However, according to Eq. (4), the actual power of the emitted radiation $P=$ $\gamma^{-2} S \theta^{2} D^{2} \Delta \nu$, which for parameters given above yields approximately

$$
P \sim \gamma_{2}^{-4} 10^{24} \operatorname{ergs~s}^{-1}
$$

much less than the luminosity derived directly from the observed duration of giant subpulses.

Melikidze, Gil \& Pataraya (2000) proposed a model for generation of the coherent pulsar radio emission, based on the idea of the polar gap discharging via a number of localized sparks. The sparking phenomenon creates a succession of plasma clouds moving along dipolar magnetic field lines. The overlapping of charged particles with different energies from adjacent clouds ignites a strong Langmuir turbulence via a two-stream instability. In the pulsar magnetospheric conditions this turbulence is subject to the modulational instability, which leads to the formation of "bunch-like" charged solitons capable of generating the coherent curvature emission at radio-wavelengths $\lambda$. Thus, the condition $\delta l=\lambda<\Delta=\rho / \gamma^{3}=\left(\rho_{8} / \gamma_{2}^{3}\right) 10^{2}$ is naturally satisfied at the centimeter wavelengths (note that according to Eq. (3), $\delta l<60 \mathrm{~cm}$ ). Additionally, the angular extent $\Delta \psi$ of the bundle of dipolar field lines associated with a given spark should not exceed $\theta=\gamma^{-1}$. It is easy to show that in the Crab pulsar $\Delta \psi \sim 0.001<\gamma^{-1}$ (see Gil \& Sendyk 2000 for details).

Melikidze et al. (2000) calculated the power of soliton curvature radiation in the vacuum approximation. Gil, Lyubarski \& Melikidze (2004) reconsidered the curvature radiation of a point-like charge moving through an electron-positron plasma, demonstrating that the radiation is suppressed by a factor $\sim 10^{-2}-10^{-3}$ but is still at a high enough level to explain the observed luminosities. Applying the results of Melikidze et al. (2000) modified by Gil et al. (2004) to the Crab, 
we obtain that the power radiated by one soliton can be as high as

$$
L_{1} \sim 10^{22} \mathrm{ergs} \mathrm{s}^{-1}
$$

(see Eqs. [14]-[18] in Melikidze et al. 2000 for details). Comparing this with the power required for the Crab subpulses expressed by Eq. (5) one obtains $\gamma_{2} \gtrsim 3$, consistent with an estimate given by Gil et al. (2004). Inserting $\gamma_{2} \gtrsim 3$ into Eq. (3) we obtain $\rho_{8} \gtrsim 16$, meaning $r_{6}^{1 / 2} s^{-1} \sim 90$. Since $r_{6}<35$ (Kijak \& Gil 1998), this means $s<0.07$. Remembering that $s$ is a normalized polar coordinate ( 0 for the pole and 1.0 for the polar cap edge) we conclude that only those field lines originating close to the magnetic dipolar axis can be involved with the nanosecond giant subpulses. Such field lines can be aligned with the observer in the radio emission region only within a narrow range of longitudes near the fiducial plane containing both the spin and magnetic axes. It is natural to associate this plane with the peak of the pulse profile. Thus, within our model, the giant nanopulses should occur only within a narrow range of longitudes near the peak of the radio profile. Accordingly, both ordinary and giant pulses are related to the inner gap sparking activity and originate at relatively low altitudes, contrary to the suggestion that they both arise in the outer gap region.

Curvature radiation from the particular soliton(s) can be observationally distinguished only if they are more powerful than other solitons. This implies that the sparking event associated with giant subpulses is more energetic than an average one. Both the luminosity and the characteristic frequency of the soliton curvature radiation strongly depend on $\gamma$, which in turn depends on the accelerating potential drop above the polar cap. Since generation of giant pulses requires sparking events occurring under an exceptionally high potential drop, it is not difficult to understand why only four pulsars are known to exhibit this phenomenon. Interestingly, those pulsars are distinguished not only by their brightness, but also by extremely high values of the "complexity parameter" $a=\left(r_{p} / h\right)$, where $r_{p}$ is the polar cap radius and $h$ is the polar gap height $(a=$ 250, 200, 30 and 25 in PSRs B0531+21, B0540-69, B1821-24 and B1937+21, respectively). Since the accelerating potential drop $\Delta V \propto h^{2}$ and $h<r_{p}$, pulsars with very high values of $a>>1$ must have a large reservoir of the maximum available potential drop over the actual potential drop $\left(\Delta V_{\max } / \Delta V=a^{2}\right)$. This reservoir can be occasionally used to create exceptionally energetic spark(s).

Acknowledgments. This work was supported in part by the Polish State Committee for Scientific Research Grant 2 P03D 00819.

\section{References}

Gil, J., \& Sendyk, M. 2002, ApJ, 541, 351

Gil, J., Lyubarsky, Y., \& Melikidze, G. I. 2004, ApJ, 600, 872

Hankins, T. H., Kern, J. S., Weatherall, J. C., \& Eilek, J. A. 2003, Nature, 422, 141

Melikidze, G. I, Gil, J., \& Pataraya, A. D. 2000, ApJ, 544, 1081

Kijak, J., \& Gil, J. 1998, MNRAS, 299, 855

McLauglin, M. A., \& Cordes, J. M. 2003, ApJ, 596, 982 